\title{
Relationship Between Chronic Periodontitis and Lower Urinary Tract Symptoms/Benign Prostatic Hyperplasia
}

\author{
Hana Hyun ${ }^{1}$, Yeon Won Park², Young Chol Kwon ${ }^{1}$, Byeng Ken Cho ${ }^{3}$, Jun Ho Lee ${ }^{4}$ \\ ${ }^{1}$ Department of Dentistry, National Police Hospital, Seoul, Korea \\ ${ }^{2}$ Department of Urology, National Police Hospital, Seoul, Korea \\ ${ }^{3}$ Department of Preventive Dentistry, Dankook University College of Dentistry, Cheonan, Korea \\ ${ }^{4}$ Department of Urology, Nowon Eulji Medical Center, Eulji University, Seoul, Korea
}

Purpose: We investigated the relationship between lower urinary tract symptoms (LUTS)/benign prostatic hyperplasia (BPH) and chronic periodontitis $(\mathrm{CP})$.

Methods: A total of 103 middle-aged men who had received a health checkup were included. All participant data were prospectively collected. CP was defined as a $30 \%$ increase in the number of probed sites with a clinical attachment level of $\geq 4 \mathrm{~mm}$ among all probed sites. LUTS/BPH were assessed using transrectal ultrasonography, the International Prostate Symptom Score (IPSS), uroflowmetry, and postvoiding residual urine volume.

Results: The median age, IPSS, prostate volume, and maximal flow rate were 55.0 years, $9.0,29.0 \mathrm{~mL}$, and $20.0 \mathrm{~mL} / \mathrm{sec}$, respectively. In addition, the prevalence of CP was $27.2 \%$. The IPSS total, IPSS voiding, IPSS storage, and quality of life (QoL) scores were significantly higher in patients with CP (median [interquartile range, IQR]-IPSS total: 8.0 [5.0-13.5] vs. 12.0 [7.5-20.5], $\mathrm{P}=0.004$; IPSS voiding: 5.0 [2.0-9.0] vs. 8.5 [4.0-15.0], $\mathrm{P}=0.002$; IPSS storage: 3.0 [2.0-5.0] vs. 4.0 [3.0-6.0], $\mathrm{P}=0.021$; $\mathrm{QoL}$ : $2.0[1.0-3.0]$ vs. $3.0[2.0-4.0], \mathrm{P}=0.015$ ). Additionally, the average flow rate was significantly lower in patients with $\mathrm{CP}$ (median [IQR] (mL/sec): 9.0 [8.0-13.0] vs. 8.0 [6.0-11.0], $\mathrm{P}=0.047)$. After adjustment for age, testosterone level, prostate volume, glucose level, cholesterol level, and waist circumference, the IPSS total and voiding scores were significantly and positively related to CP (IPSS total: odds ratio [OR], 1.141; 95\% confidence interval [CI], 1.045-1.245; P=0.003; IPSS voiding: OR, 1.243; 95\% CI, 1.092-1.415; $\mathrm{P}=0.001)$.

Conclusions: Our data suggest that LUTS/BPH is significantly related to CP.

Keywords: Lower urinary tract symptoms; Benign prostatic hyperplasia; Chronic periodontitis

- Research Ethics: This study was approved by the Institutional Review Board (IRB) of the National Police Hospital (IRB No. 11100176-201909HR-009).

- Conflict of Interest: No potential conflict of interest relevant to this article was reported.

\section{INTRODUCTION}

Chronic periodontitis (CP) is an infectious disease resulting in inflammation within the supporting tissue of the teeth, progressive attachment, and bone loss [1]. CP is a highly prevalent dis- ease, and a nationwide cohort study in Korea reported that among a total of $1,025,340$ samples, $31.3 \%$ of the associated patients were diagnosed with periodontitis [2]. Recently, a relationship between $\mathrm{CP}$ and systemic diseases including cardiovascular disease [3], metabolic syndrome [4,5], and endothelial

Corresponding author: Jun Ho Lee (D) https://orcid.org/0000-0003-3567-0816 Department of Urology, Nowon Eulji Medical Center, Eulji University, 68 Hangeulbiseok-ro, Nowon-gu, Seoul 01830, Korea Email: sinbanpolee@gmail.com

Submitted: March 1, 2020 / Accepted after revision: June 26, 2020 
dysfunction [6] has been reported.

Benign prostatic hyperplasia (BPH) refers to voiding dysfunction resulting from prostate enlargement and bladder outlet obstruction [7]. BPH is caused by nonmalignant, unregulated growth of the prostate gland, and it is a major cause of lower urinary tract symptoms (LUTS) [8]. BPH is highly prevalent in older men, and the prevalence was $27.7 \%$ according to a community-based study [9]. Interestingly, recent data showed that LUTS/BPH was related to CP. However, scant data are currently available, and these data are limited by the definition of both $\mathrm{CP}$ and LUTS/BPH by using only a simple questionnaire or the absence of adjustment for potential confounding factors such as age, obesity, and metabolic factors. The relationship between $\mathrm{CP}$ and LUTS/BPH might be an important issue because both $\mathrm{CP}$ and LUTS/BPH are highly prevalent diseases. Therefore, we conducted this study to investigate the relationship between $\mathrm{CP}$ and LUTS/BPH in order to gain a better understanding of the pathophysiology of both CP and LUTS/BPH.

\section{MATERIALS AND METHODS}

\section{Subjects}

From September 2017 to August 2019, 108 men in their 40s or 50s received LUTS/BPH and CP health check-ups. We collected all participant data prospectively.

\section{Periodontal Examination}

According to the clinical criteria proposed by the World Workshop on Periodontics in 1999, CP was defined as a 30\% increase in the number of probed sites with a clinical attachment level (CAL) of $\geq 4 \mathrm{~mm}$ among all probed sites [10].

Each patient underwent periodontal examinations. We measured the plaque index, bleeding upon probing, probing depth, and CAL. Bleeding upon probing was assessed by using the bleeding point index. The probing depth was defined as the distance between the gingival margin and the deepest aspect of the pocket. The CAL was defined as the distance between the cementoenamel junction of the tooth and the deepest aspect of the pocket. A periodontal measurement was made on 6 surfaces per tooth (mesiobuccal, midbuccal, distobuccal, mesiolingual, midlingual, and distolingual) of all teeth except the third molars using a Willams probe (PCP-15, Hu-Friedy, Chicago, IL, USA). A single periodontist and 2 well-trained dentists who were blinded to the urological data performed all periodontal examination. Before the start of the study, the periodontal ex- aminer was trained on the periodontal examination standard. In this study, we used only the CAL parameter to investigate the relationship between $\mathrm{CP}$ and LUTS/BPH because other parameters were not significantly related to LUTS/BPH in our pilot study.

\section{Assessment of LUTS/BPH}

The urinary flow rate was assessed by uroflowmetry (Medtronic Inc., Minneapolis, MN, USA) and postvoid residual urine volume was measured using transabdominal ultrasonography (UltraView 800, BK Medical, Amsterdam, The Netherlands). We discarded the uroflowmetry data when the voided was less than $150 \mathrm{~mL}$ [11]. We also used the validated Korean version of the International Prostate Symptom Score (IPSS). We measured the total prostate volume using transrectal ultrasonography (UltraView 800, BK Medical, Herlev, Denmark). All total prostate volume measurements were made by the same sonographer. We calculated the total prostate volume according to previous methods [12].

$\mathrm{BPH}$ was defined as IPSS $\geq 8$ [13], maximal flow rate $<15$ $\mathrm{mL} / \mathrm{sec}$ [14], and prostate gland volume $\geq 30 \mathrm{~mL}$ [13].

\section{Anthropometry and Biochemical/Hormonal Assay}

The waist circumference $(\mathrm{cm})$ was measured to the nearest 0.1 $\mathrm{cm}$ midway between the lowest rib and the iliac crest. Serum was drawn between 7:00 AM and 9:00 AM after an overnight fasting. The biochemical analysis measurements included prostate-specific antigen, fasting serum glucose level, and total cholesterol level. Serum testosterone was measured via radioimmunoassay by using a kit from Cisbio Bioassays, Inc. (Parc Marcel Boiteux, Codolet, France). The intra-assay coefficients of variation for all assays were less than $9 \%$, and the interassay coefficients of variation were less than $12 \%$. For each assay, all samples from each subject were used in the same assay run.

\section{Potential Confounders}

Age [15], diabetes mellitus (DM) [15], hyperlipidemia [16], and obesity [15] are known to be risk factors for CP. Additionally, some reports demonstrated that low testosterone levels were associated with CP [17]. Age, DM, hyperlipidemia, hormonal imbalance, and obesity are also known as risk factors for LUTS/ BPH [18]. Therefore, we adjusted these confounders when elucidating the relationship between LUTS/BPH and CP.

Obesity was assessed by waist circumference. DM was defined as fasting blood glucose $\geq 126 \mathrm{mg} / \mathrm{dL}$, or use of antidia- 
betic medication, or insulin [19]. Hyperlipidemia was defined as total cholesterol $>240 \mathrm{mg} / \mathrm{dL}$ or use of antihypercholesterolaemic medication [19].

\section{Statistical Analysis}

We excluded the following subjects from the study: (1) those who were administered certain drugs, including antipsychotics, alpha blockers, 5-alpha reductase inhibitors, or phosphodiesterase-5 inhibitors; (2) those who underwent surgery or were hospitalized in the last 3 months; and (3) those with pyuria. Finally, 103 subjects were included in this study.

First, we evaluated whether there were any differences in the prostate volume, maximal flow rate, average flow rate, residual urine volume, IPSS total score, IPSS voiding subscore, IPSS storage score, IPSS QoL score, CAL measurements and the percentage of cases of prostate volume $\geq 30 \mathrm{~mL}$, maximal flow rate $<15 \mathrm{~mL} / \mathrm{sec}$, maximal flow rate $<10 \mathrm{~mL} / \mathrm{sec}$, IPSS total $\geq 8$, IPSS total $\geq 20$, and $\mathrm{BPH}$ or $\mathrm{CP}$ according to the presence of $\mathrm{CP}$ or $\mathrm{BPH}$ using the Mann-Whitney U-test or Fisher exact test Finally, we tested whether there was a relationship between CP and the prostate volume, maximal flow rate, average flow rate, residual urine volume, IPSS total score, IPSS voiding score, IPSS storage score, or IPSS QoL score after adjusting for confounding factors using logistic regression analysis.

All tests were 2-sided, with statistical significance set at $\mathrm{P}<0.05$. Analyses were conducted using $\mathrm{R}$ ver. 2.13.1 ( $\mathrm{R}$ Foundation for Statistical Computing, Vienna, Austria).

\section{RESULTS}

\section{Patient Characteristics}

The characteristics of the study subjects are shown in Table 1. The median age, total prostate volume, IPSS total score, and maximal flow rate were 55.0 years, $29.0 \mathrm{~mL}, 9.0$, and $20.0 \mathrm{~mL} /$ $\mathrm{sec}$, respectively. In addition, the prevalence of CP was $27.2 \%$ (Table 1).

\section{Relationships Between CP and LUTS/BPH}

In subjects with $\mathrm{CP}$, the age, prostate volume, residual urine volume, IPSS total score, IPSS voiding score, IPSS storage score, and IPSS quality of life (QoL) score were higher, while maximal flow rate and average flow rate were lower.

In addition, the percentage of cases of prostate volume $\geq 30$ $\mathrm{mL}$, maximal flow rate $<15 \mathrm{~mL} / \mathrm{sec}$, maximal flow rate $<10$ $\mathrm{mL} / \mathrm{sec}$, IPSS total $\geq 8$, IPSS total $\geq 20$, and $\mathrm{BPH}$ (IPSS $\geq 8$, maximal flow rate $<5 \mathrm{~mL} / \mathrm{sec}$, and prostate gland volume $\geq 30$ $\mathrm{mL}$ ) were significantly greater in the subjects with CP.

Moreover, there were significant differences in the age, average flow rate, IPSS total score, IPSS voiding score, IPSS storage score, IPSS QoL score, and the percentage of cases of IPSS total $\geq 20$ and $\mathrm{BPH}$ (IPSS $\geq 8$, maximal flow rate $<15 \mathrm{~mL} / \mathrm{sec}$, and prostate gland volume $\geq 30 \mathrm{~mL}$ ) between the 2 groups, which suggests that men with CP have more severe LUTS/BPH (Table $1)$.

In the subjects with BPH, CAL mean and the percentage of cases of CAL $\geq 4 \mathrm{~mm}$ and $\mathrm{CP}$ were significantly greater, which suggests that men with BPH had more chance of CP (Table 1). After adjusting for confounding factors, the IPSS total score, IPSS voiding score, and IPSS QoL were significantly, positively, and independently related to $\mathrm{CP}$, which means that the presence of CP is significantly, positively, and independently related to severe LUTS/BPH (Table 2).

\section{DISCUSSION}

In this study, we found that the IPSS and the percentage of cases of IPSS total $\geq 20$ or BPH were significantly higher and the average flow rate was significantly lower in men with $\mathrm{CP}$ and that the IPSS total, voiding, and QoL score were significantly, positively, and independently related to $\mathrm{CP}$, all of which suggest that LUTS/BPH severity is increased in men with CP.

The severity of LUTS is best measured using subjective symptom indices such as the IPSS questionnaire and objective diagnostic tools such as uroflowmetry, postvoid residual urine volume, and prostate size assessed by transrectal ultrasound [20]. In this study, we evaluated LUTS/BPH using all of these methods, which are widely used in urological clinical practice based on clinical guidelines [11].

In terms of IPSS questionnaires, Matsumoto et al. [21] reported the positive relevance of CP to urgency and a weak stream in their subanalysis of IPSS. However, there was no significant association of CP with the IPSS voiding score or IPSS storage score. In our study, we found significant associations of $\mathrm{CP}$ with the IPSS voiding score and IPSS storage score. Furthermore, there were significant associations of CP with the IPSS voiding score after adjustment for confounding factors. These discrepancies might be attributable to the limited number of subjects in the study of Matsumoto et al. (data from 88 male participants) and relatively young age of their participants compared to our participants ( 50.9 years vs. 55.0 years). Additional- 


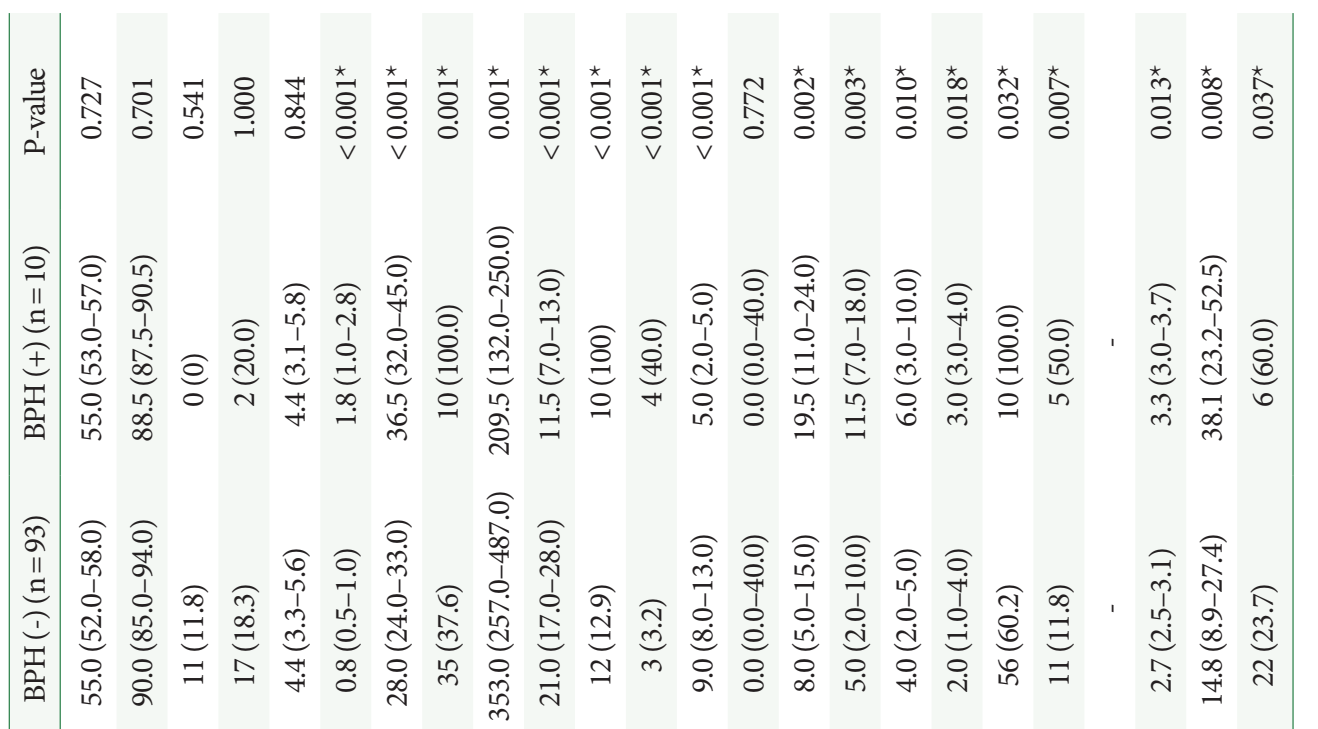

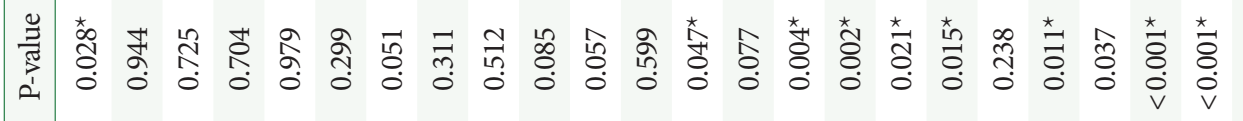

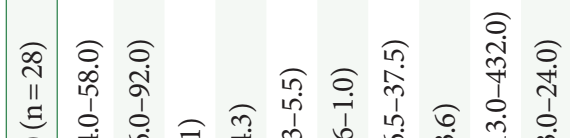

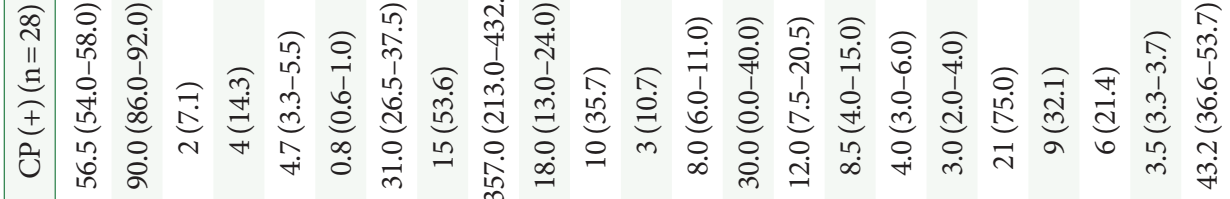

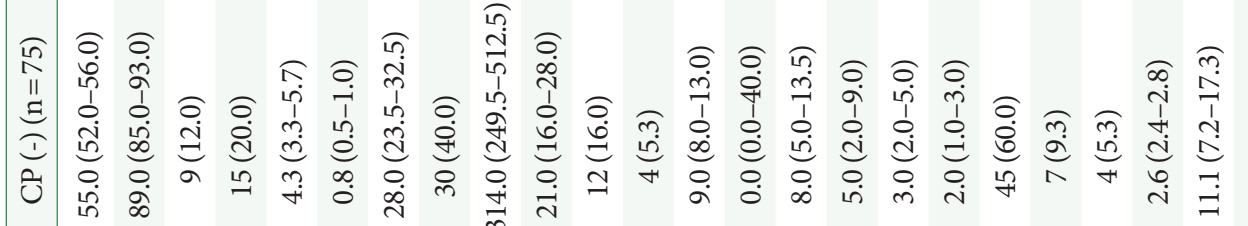

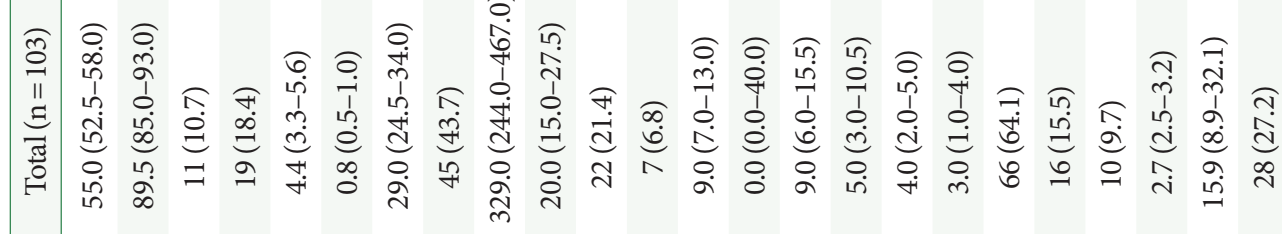

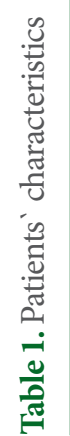

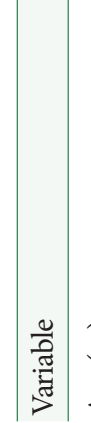

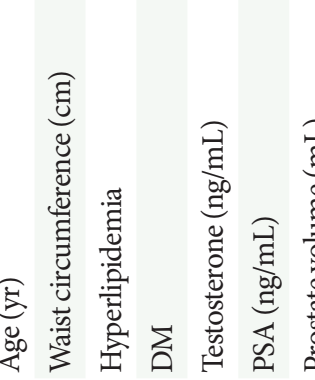

苾

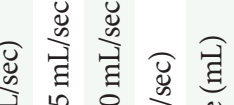

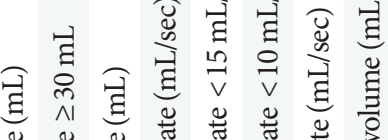

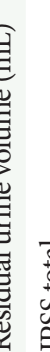

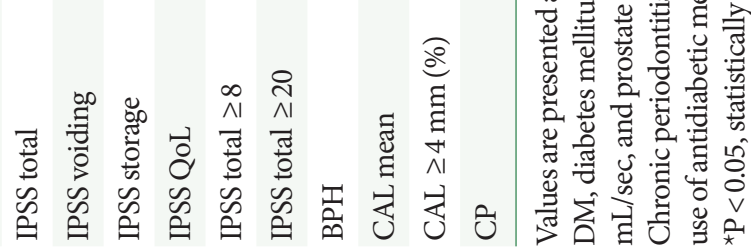


Table 2. Adjusted ORs of LUTS/BPH parameters for CP

\begin{tabular}{|c|c|c|}
\hline Variable & OR $(95 \% \mathrm{CI})$ & P-value ${ }^{\dagger}$ \\
\hline Prostate volume $(\mathrm{mL})^{\mathrm{a}}$ & $1.024(0.960-1.093)$ & 0.471 \\
\hline Maximal flow rate $\left.(\mathrm{mL} / \mathrm{sec})^{\mathrm{b}}\right)$ & $0.967(0.899-1.040)$ & 0.366 \\
\hline Average flow rate $(\mathrm{mL} / \mathrm{sec})^{\mathrm{b})}$ & $0.939(0.793-1.113)$ & 0.470 \\
\hline Residual urine volume $\left.(\mathrm{mL})^{\mathrm{b}}\right)$ & $1.004(0.995-1.012)$ & 0.374 \\
\hline IPSS total ${ }^{c}$ & $1.141(1.045-1.245)$ & $0.003^{*}$ \\
\hline IPSS voiding ${ }^{c}$ & $1.243(1.092-1.415)$ & $0.001^{\star}$ \\
\hline IPSS storage $e^{c)}$ & $1.219(0.997-1.489)$ & 0.054 \\
\hline IPSS QoL ${ }^{c)}$ & $1.676(1.124-2.500)$ & $0.011^{\star}$ \\
\hline
\end{tabular}

Chronic periodontitis was defined as a $30 \%$ increase in the number of probed sites with a CAL of $\geq 4 \mathrm{~mm}$ among all probed sites.

OR, odds ratio; LUTS, lower urinary tract symptoms; $\mathrm{BPH}$, benign prostatic hyperplasia; CP, chronic periodontitis; IPSS, International Prostate Symptom Score; QoL, quality of life.

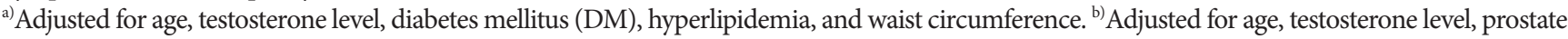
volume, DM, hyperlipidemia, waist circumference, and voided volume during uroflowmetry. ${ }^{c}$ Adjusted for age, testosterone level, prostate volume, $\mathrm{DM}$, hyperlipidemia, and waist circumference. ${ }^{\dagger}$ Logistic regression analysis.

${ }^{\star} \mathrm{P}<0.05$, statistically significant differences.

ly, Matsumoto et al. [21] used a simple questionnaire, the chronic periodontal disease self-checklist, to define $\mathrm{CP}$, which is a definite drawback to defining CP. Considering that the number of participants is related to the power of the data, that age is the most important factor for developing LUTS/BPH, and that the assessment of $\mathrm{CP}$ was more appropriate in our study, the aforementioned factors could explain the very different results between Matsumoto et al's and our data.

With regard to prostate volume, we first assessed the prostate volume in studies on the relationship between CP and LUTS/ $\mathrm{BPH}$. Boland et al. [22] reported an association between BPH and $\mathrm{CP}$ with the largest number of participants. However, they used International Classification of Diseases (ICD)-9 codes to represent medical conditions. It is well known that ICD-9 codes have low specificity [23]. Additionally, these data are limited by a lack of data on prostate size. Because the prostate volume is an important part of the diagnosis and pathogenesis of $\mathrm{BPH}$, our data are valuable. In our study, the total prostate volume in men with $\mathrm{CP}$ was larger than that in men without $\mathrm{CP}$, and the difference was nearly statistically significant. (28 mL vs. $31 \mathrm{~mL}$, $\mathrm{P}=0.051)$. Further study is needed to investigate the relationship between $\mathrm{CP}$ and prostate volume.

In regard to uroflowmetry, we first used this method to examine the strength of the urinary stream as identified in reports about the relationship between CP and LUTS/BPH. Our findings demonstrated that the average flow rate was significantly lower in men with $\mathrm{CP}$, which suggests that a weak urinary stream is related to CP. Uroflowmetry is one of the most commonly used examinations for the objective measurement of voiding difficulty. Additionally, uroflowmetry is a time-consuming method to conduct, because participants need to hold their urine for approximately 2 to 3 hours. Therefore, our results have significant clinical implications. Our data showed that LUTS/BPH was significantly related to $\mathrm{CP}$ using objective assessment methods (uroflowmetry) as well as subjective methods (IPSS).

The reason that $\mathrm{CP}$ is related to LUTS/BPH is unclear. Additionally, in this clinical cross-sectional setting, it is difficult to determine the pathophysiology underlying these relationships. However, we can assume the reasons from previous data.

Metabolic syndrome refers to a cluster of cardiovascular risk factors, including atherogenic dyslipidemia, elevated blood pressure, obesity, hypertension, elevated glucose, a prothrombotic state, and a proinflammatory state [24]. Metabolic syndrome is a risk factor for both CP [25] and LUTS/BPH [26,27], which means that both $\mathrm{CP}$ and LUTS/BPH share metabolic syndrome as a risk factor. We believe that this similarity is an explanation of the relationship between CP and LUTS/BPH.

The other possible explanation is the direct dissemination of oral pathogens to the prostate. There is a report that oral pathogens in subgingival plaque have simultaneously been detected in the prostate fluid of men [28]. In addition, it has been suggested that bacterial prostatitis is related to the development of BPH $[29,30]$. Therefore, prostatitis from oral pathogens might 
be the cause of the association between CP and LUTS/BPH.

Systemic inflammation could be another explanation of the relationship. It has been reported that periodontal diseases are related to markers of systemic inflammation, such as interleukin (IL)-1, IL-2, tumor necrosis factor-alpha, and C-reactive protein. It has also been suggested that systemic inflammation affects voiding dysfunction [31]. Therefore, systemic inflammation induced by $\mathrm{CP}$ might exacerbate the voiding difficulties found in this study.

The present study has several limitations. First, this cross-sectional study could not be used to infer causality. Second, we could not adjust genetics and inflammations components as risk factors, both of which are one of important risk factors [8]. Additionally, this study might have a potential selection bias because we used a single institutional data. However, despite these limitations, we believe that the results of this study are highly relevant because among studies evaluating LUTS/BPH and $\mathrm{CP}$, this study is the first to combine the use of transrectal ultrasound, uroflowmetry, and IPSS and the first to adjust for potential confounding factors including age and metabolic syndrome components.

The present results show that CP is closely related to LUTS/ $\mathrm{BPH}$ in men in their 40s and 50s. Future investigations should explore the temporal relationship between CP and BPH/LUTS. To confirm this, a prospective, longitudinal data set would be needed.

\section{AUTHOR ORCID}

$\begin{array}{ll}\text { Hana Hyun } & 0000-0002-3630-049 X \\ \text { Yeon Won Park } & 0000-0003-0751-8892 \\ \text { Young Chol Kwon } & 0000-0003-2940-4992 \\ \text { Byeng Ken Cho } & 0000-0002-3140-2294 \\ \text { Jun Ho Lee } & 0000-0003-3567-0816\end{array}$

\section{AUTHOR CONTRIBUTION STATEMENT}

- Conceptualization: BKC, JHL

- Data curation: HH, YWP, YCK, JHL

- Formal analysis: JHL

- Funding acquisition: JHL

- Methodology: JHL

- Project administration: JHL

- Visualization: JHL

-Writing-original draft: $\mathrm{HH}$, JHL
-Writing-review \& editing: JHL

\section{REFERENCES}

1. 1999 International International Workshop for a Classification of Periodontal Diseases and Conditions. Papers. Oak Brook, Illinois, October 30-November 2, 1999. Ann Periodontol 1999;4:i, 1-112.

2. Lee JH, Lee JS, Park JY, Choi JK, Kim DW, Kim YT, et al. Association of lifestyle-related comorbidities with periodontitis: a nationwide cohort study in Korea. Medicine (Baltimore) 2015;94:e1567.

3. Dietrich T, Garcia RI. Associations between periodontal disease and systemic disease: evaluating the strength of the evidence. J Periodontol 2005;76(11 Suppl):2175-84..

4. D’Aiuto F, Sabbah W, Netuveli G, Donos N, Hingorani AD, Deanfield J, et al. Association of the metabolic syndrome with severe periodontitis in a large U.S. population-based survey. J Clin Endocrinol Metab 2008;93:3989-94.

5. Li P, He L, Sha YQ, Luan QX. Relationship of metabolic syndrome to chronic periodontitis. J Periodontol 2009;80:541-9.

6. Higashi Y, Goto C, Jitsuiki D, Umemura T, Nishioka K, Hidaka T, et al. Periodontal infection is associated with endothelial dysfunction in healthy subjects and hypertensive patients. Hypertension 2008;51:446-53.

7. Lee JH, Park YW, Lee SW. The relationships between thyroid hormone levels and lower urinary tract symptoms/benign prostatic hyperplasia. World J Mens Health 2019;37:364-71.

8. Ko IG, Hwang L, Jin JJ, Kim SH, Han JH, Jeon JW, et al. Add-on therapy with the $\alpha$-blockers tamsulosin and naftopidil improves voiding function by enhancing neuronal activity in prostatic hyperplasia rats. Int Neurourol J 2018;22:20-9.

9. Lee HL, Seo JW, Kim WJ. The prevalence of benign prostatic hyperplasia: community-based study in Chungbuk province. Korean J Urol 1999;40:1500-5.

10. Eke PI, Genco RJ. CDC Periodontal Disease Surveillance Project: background, objectives, and progress report. J Periodontol 2007;78 (7 Suppl):1366-71.

11. Gratzke C, Bachmann A, Descazeaud A, Drake MJ, Madersbacher S, Mamoulakis C, et al. EAU Guidelines on the assessment of nonneurogenic male lower urinary tract symptoms including benign prostatic obstruction. Eur Urol 2015;67:1099-109.

12. Rhodes T, Girman CJ, Jacobsen SJ, Roberts RO, Guess HA, Lieber $\mathrm{MM}$. Longitudinal prostate growth rates during 5 years in randomly selected community men 40 to 79 years old. J Urol 1999; 161:1174-9.

13. Huh JS, Kim YJ, Kim SD. Prevalence of benign prostatic hyperpla- 
sia on Jeju island: analysis from a cross-sectional community-based survey. World J Mens Health 2012;30:131-7.

14. Park YW, Min SK, Lee JH. Relationship between lower urinary tract symptoms/benign prostatic hyperplasia and metabolic syndrome in Korean men. World J Mens Health 2012;30:183-8.

15. Genco RJ, Borgnakke WS. Risk factors for periodontal disease. Periodontol 2000 2013;62:59-94.

16. Lianhui Y, Meifei L, Zhongyue H, Yunzhi F. Association between chronic periodontitis and hyperlipidemia: a Meta-analysis based on observational studies. Hua Xi Kou Qiang Yi Xue Za Zhi 2017; 35:419-26.

17. Kellesarian SV, Malmstrom H, Abduljabbar T, Vohra F, Kellesarian $\mathrm{TV}$, Javed F, et al. Low testosterone levels in body fluids are associated with chronic periodontitis. Am J Mens Health 2017;11:44353.

18. Calogero AE, Burgio G, Condorelli RA, Cannarella R, La Vignera S. Epidemiology and risk factors of lower urinary tract symptoms/benign prostatic hyperplasia and erectile dysfunction. Aging Male 2019;22:12-9.

19. Korean Diabetes Association. Diabetes fact sheet in Korea 2016. Seoul: Korean Diabetes Association, 2016. p. 2-8.

20. Sarma AV, Wei JT. Clinical practice. Benign prostatic hyperplasia and lower urinary tract symptoms. N Engl J Med 2012;367:248-57.

21. Matsumoto S, Matsuda M, Takekawa M, Okada M, Hashizume K, Wada N, et al. Association between chronic periodontal disease and lower urinary tract symptoms in both sexes. Low Urin Tract Symptoms 2015;7:17-21.

22. Boland MR, Hripcsak G, Albers DJ, Wei Y, Wilcox AB, Wei J, et al. Discovering medical conditions associated with periodontitis using linked electronic health records. J Clin Periodontol 2013;40:474-82.

23. Birman-Deych E, Waterman AD, Yan Y, Nilasena DS, Radford MJ, Gage BF. Accuracy of ICD-9-CM codes for identifying cardiovascular and stroke risk factors. Med Care 2005;43:480-5.

24. Eckel RH, Alberti KG, Grundy SM, Zimmet PZ. The metabolic syndrome. Lancet 2010;375:181-3.

25. Gurav AN. The association of periodontitis and metabolic syndrome. Dent Res J (Isfahan) 2014;11:1-10.

26. Kupelian V, McVary KT, Kaplan SA, Hall SA, Link CL, Aiyer LP, et al. Association of lower urinary tract symptoms and the metabolic syndrome: results from the Boston area community health survey. J Urol 2013;189(1 Suppl):S107-14.

27. Park YW, Kim SB, Kwon H, Kang HC, Cho K, Lee KI, et al. The relationship between lower urinary tract symptoms/benign prostatic hyperplasia and the number of components of metabolic syndrome. Urology 2013;82:674-9.

28. Estemalik J, Demko C, Bissada NF, Joshi N, Bodner D, Shankar E, et al. Simultaneous detection of oral pathogens in subgingival plaque and prostatic fluid of men with periodontal and prostatic diseases. J Periodontol 2017;88:823-9.

29. Khan FU, Ihsan AU, Khan HU, Jana R, Wazir J, Khongorzul P, et al. Comprehensive overview of prostatitis. Biomed Pharmacother 2017;94:1064-76.

30. Fibbi B, Penna G, Morelli A, Adorini L, Maggi M. Chronic inflammation in the pathogenesis of benign prostatic hyperplasia. Int $\mathrm{J}$ Androl 2010;33:475-88.

31. Lloyd GL, Marks JM, Ricke WA. Benign prostatic hyperplasia and lower urinary tract symptoms: what is the role and significance of inflammation? Curr Urol Rep 2019;20:54. 